\title{
A MULTI-PURPOSE CULTURAL HERITAGE DATA PLATFORM FOR 4D VISUALIZATION AND INTERACTIVE INFORMATION SERVICES
}

\author{
C. Ioannidis *, S. Verykokou, S. Soile, A.-M. Boutsi \\ Laboratory of Photogrammetry, School of Rural \& Surveying Engineering, National Technical University of Athens, Greece
}

Commission IV, WG IV/9

KEY WORDS: Geospatial data, documentation, visualization, 4D modelling, cultural heritage

\begin{abstract}
:
The already arduous task of collecting, processing and managing heterogeneous cultural heritage data is getting more intense in terms of indexing, interaction and dissemination. This paper presents the creation of a 4D web-based platform as a centralized data hub, moving beyond advanced photogrammetric techniques for 3D capture and multi-dimensional documentation. Precise metric data, generated by a combination of image-based, range and surveying techniques, are spatially, logically and temporally correlated with cultural and historical resources, in order to form a critical knowledge base for multiple purposes and user types. Unlike conventional information systems, the presented platform, which adopts a relational database model, has the following front-end functionalities: (i) visualization of high-resolution 3D models based on distance dependent Level of Detail (LoD) techniques; (ii) web Augmented Reality; and (iii) interactive access and retrieval services. Information deduced from the developed services is tailored to different target audiences: scientific community, private sector, public sector and general public. The case study site is the UNESCO world heritage site of Meteora, Greece, and particularly, two inaccessible huge rocks, the rock of St. Modestos, known as Modi, and the Alyssos rock.
\end{abstract}

\section{INTRODUCTION}

Heterogeneous data management practices in the heritage sector are very important and demand increasing attention. The geometric documentation of historical and archaeological sites entails metric precision, 3D modeling in high resolution and integration of diversified information. Data volume is getting bigger, the variety of data sources is getting wider and the deriving value is getting even greater. These high-level requirements evolve in line with technological developments in 3D data acquisition, processing and visualization. In the context of the aforementioned requirements and technological progress, much research has been conducted using various data sources, like data from close-range photogrammetry (Dall'Asta et al., 2016) or LiDAR data (Cao et al., 2018), or implementing reverse engineering methods (Ioannidis et al., 2019), in order to generate accurate $3 \mathrm{D}$ representations using computer vision algorithms. In addition, the third dimension is necessary for web-based information systems (López et al., 2019), immersive gaming environments (Cipriani et al., 2019) and multiresolution visualization platforms (Agugiaro et al., 2011). Storage, query and archiving workflows may vary from relational database systems and NoSQL approaches suited to big data, to semantic-based layouts (Ben Ellefi et al., 2019; ElMahgary et al., 2020).

The generation of 3D models at different scales has already been addressed by the research community (Salonia et al., 2009). Also, structures for geographic information that support smooth zoom and may handle both 2D and 3D data have been proposed (van Oosterom and Meijers, 2011; 2014) and structures that implement continuous-scale 3D visualization based on a detail-increment model that avoids the disadvantages of a static Level of Detail (LoD) model have been introduced (Ai et al., 2019). Furthermore, the concept of multiple dimensions, has already been implemented in various systems, e.g., by Ohori et al. (2015), who introduced scale as the fourth dimension, and by Ioannidis et al. (2015), who introduced time and scale as additional dimensions within a 5D land information system.

The design of a methodology for 4D visualization (i.e., visualization of $3 \mathrm{D}$ models at various scales), indexing and sharing of multi-dimensional models and other kinds of heterogeneous data is the overview of the presented work. This paper introduces the scope, progress and outreach of the ongoing research project "Information System for Multi-Level Documentation of Religious Sites and Historic Complexes: METEORA" (www.meteora.net.gr). The project promotes an interdisciplinary approach to heritage. It considers how critical information for the preservation, diagnosis and maintenance of archaeological sites can be depicted in 3D spatial models and disseminated to the end users. The project consortium consists of surveying engineers, electrical engineers, theologians, geologists, architects and scientists with confirmed skills about surveys, who are members of three academic institutes and three companies in Greece. The case study of the project is the geometric documentation of two inaccessible rocks of the archaeological site of Meteora, Greece, i.e., the rock of St. Modestos, known as Modi, on top of which ruins of the old monastery of St. Modestos are located, and the rock Alyssos, where the monastery of the Chain of Apostle Peter used to exist. These rocks are of great height (about $200 \mathrm{~m}$ ) and the ascent to the rocks is of increased difficulty. Considering the morphological peculiarities of the case study and the plan of 3D surveying and mapping a region of $10 \mathrm{~km}^{2}$, the proposed workflow can be tailored to every historical complex of a largeextent and difficult topography.

According to the overall workflow of the METEORA project, data collection and processing take place through fast and automated techniques. Large datasets of images from various 
sources along with terrestrial measurements and georeferencing information from onboard sensors are used for the creation of $3 \mathrm{D}$ models of the two rocks and the surrounding space in different resolutions. The $3 \mathrm{D}$ models at different scales will configure a $4 \mathrm{D}$ functional model, incorporating scale as the fourth dimension. The final implementation will represent a multi-component platform incorporating the following functionalities:

(i) Flexible back-end infrastructure: Storing, querying and accessing interoperable data based on a relational database management system (RDBMS);

(ii) Interactive information system: Collaborative data retrieval and analysis through navigation tools, annotations and customized services for various users and use cases;

(iii) Responsive 4D visualization schema: LoD representations, cashing strategy; and

(iv) Web Augmented Reality (WAR): Location-based AR for mobile devices.

Currently, the 4D platform is at a prototype phase, handling a small part of interoperable data: large-scale point clouds and 3D meshes generated through data acquired by topographic and photogrammetric techniques and a historic and cultural archive of visual and textual information. It runs on every browser that supports the WebGL API and is intended for desktops, laptops and mobile devices. Prior to the design and set-up of its backend and front-end components, main focus is given on the identification of the potential target groups and their needs.

This paper is structured as follows. Section 2 describes the system architecture and the 4D visualization pipeline. Section 3 outlines the procedures of data collection and photogrammetric processing, resulting in the presentation of the infrastructure and functionalities of the platform. Section 4 presents the user scenarios. Finally, in Section 5 conclusions and plans for future work are discussed.

\section{METHODOLOGY}

Large and heterogeneous datasets require a software architecture that prevents runtime performance problems. The proposed system is a typical client-server schema that consists of three operational levels: the presentation layer, the application layer and the persistence layer (Figure 1). It is developed independently of the operating system and the type of the display device, exploiting Node.js as a web server.

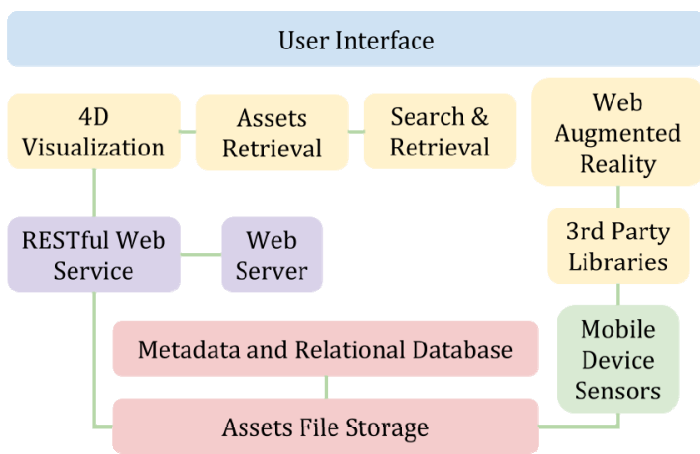

Figure 1. Browser/server architecture of the METEORA platform; each color represents a different layer: yellow for the presentation layer, purple for the application layer, red for the persistence layer and green for the Inertial Measurement Unit (IMU) of the mobile device

\subsection{Browser - Server Architecture}

The architecture's back-end system uses a flexible PostgreSQL database system for aggregating and streaming assorted data in a single, logical location. Metadata are also directly stored in the tables of the relational database and no joins are required to retrieve them. The actual resource managers are the frameworks and libraries that implement the 3D viewer, namely the Three.js 3D graphics library (Three.js, 2020) and the 3DHOP framework (Potenziani et al., 2015). The 3D scene is configured by a declarative structure with JSON (JavaScript Object Notation) encoding for its 3D content and the rest of the linked assets. All these objects are persisted to the external file storage, where they are mapped to a specific id. The relational concept of the DBMS is implemented by interpreting them with JSON functions and operators. The way data are provided to the end users is defined by the business logic of the platform, associated with the Application Layer. A web service is built to expose a REST (Representational State Transfer) API and invoke the needed database actions. Therefore, endpoints establish the client-server communication and the assets generated by the database query are easily accessible. Regarding the presentation layer, services like 4D visualization and qualitative data retrieval, depend to a great extent on the functionality of the frameworks and libraries that host the $3 \mathrm{D}$ viewer. Thus, various methods are developed to access the datasets through the 3D scene. Integrated loaders, customized to a certain format or kind of geometry, like Wavefront OBJ, Polygon File Format (PLY) and Draco files (Draco, 2020), determine the display of the assorted data. The loading method receives the assets from their file path, while textures and materials are declared as associated arrays. JavaScript event listeners are used to push all the requests for all the demanded assets and implement the provided services. Finally, the presentation layer integrates the Graphical User Interface (GUI) of the platform, namely, the layout and style of the content displayed to the end users.

The Augmented Reality (AR) module runs on top of the WebGL API and its application is hosted by a mobile device in the browser. AR.js library (AR.js, 2020) and A-Frame framework (A-Frame, 2020) intrinsically access the camera of the mobile device and its magnetic, orientation and GPS sensors. AR resources, like image markers and overlays, are stored in the repository of the same assets. The data required for the geo-location and the global pose estimation are derived from third party components via the network.

\subsection{LoD Model Viewer}

According to an approach based on LoDs, a 3D mesh is decimated into smaller partitions with less vertices/edges. Each partition represents a different LoD, which is displayed at a certain distance from the camera. As this distance gets bigger, the amount of details of the model gets smaller. Rendering is switched between the models of different LoD through smooth visual transitions. This lossless and continuous-resolution visualization technique is applied to individual large meshes, yielding optimal results regarding time and performance. The progressive streaming of multiple 3D meshes of the same object that differ in scale, detail, format or time period is a different issue. In this case, each mesh replaces the other one, while the distance from the user's point of view changes. Loading and refinement is continuous and smooth but temporal or spatial variations can be observed. In order to prevent memory leaking during dynamic loading, the various instances of the scene are retained on a cache. $3 \mathrm{D}$ models, textures and materials use the 
different loading resources provided by Three.js. Instead of implementing both a loading and a caching process, a single cache is used only for the instances of the WebGL renderer.

\section{METEORA PLATFORM}

In this section, the data collection and 3D modelling techniques applied to the case study of the Meteora site are presented and the METEORA platform is described.

\subsection{Spatial Data Collection}

The geometric documentation of cultural heritage sites with similar geomorphological characteristics and complexity to those of the Meteora site requires particular spatial data collection processes and 3D modelling techniques.

Figure 2 shows the various sources of spatial data that were used within the METEORA project. Specifically, a total of about 2200 vertical and oblique aerial images covering the Meteora site was acquired from a manned aircraft, by a NIKON $\mathrm{D} 800 \mathrm{E}$ camera. The images, taken from a flying height of about $600 \mathrm{~m}$ with respect to the top of the rocks, correspond to an average overlap of $80 \%$ and a ground sampling distance (GSD) of about $5 \mathrm{~cm}$. Each image has a size of $7360 \times 4912$ pixels, a focal length of $50 \mathrm{~mm}$ and a pixel size of $4 \mu \mathrm{m}$. The total area covered by the aerial images is about 600 ha. Also, about 4000 images depicting the giant rocks of Modi and Alyssos were acquired from a DJI Phantom IV RTK UAV, with NetworkRTK receiver on board and PPK capability, using a 20megapixel camera, from flying heights of 20 up to $30 \mathrm{~m}$. The images are accompanied by onboard GNSS data and correspond to an average overlap of $80 \%$ and a GSD of 3.5 to $5 \mathrm{~mm}$.

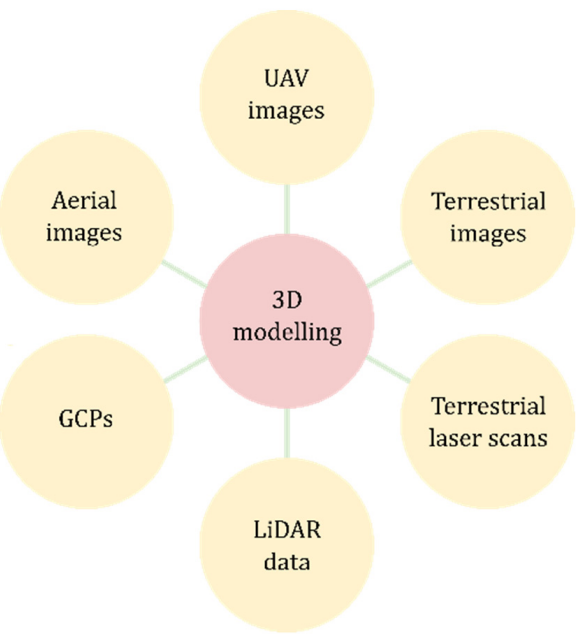

Figure 2. Spatial data collection

The acquisition of terrestrial images of all the sides of the rocks of interest, i.e., Modi and Alyssos, from a small distance was not possible, due to the inaccessible surrounding space and the particularly complex relief, characterized by cavities and protrusions at different altitudinal levels, the non-availability of any straight road and the danger involved in the use of terrestrial data collection methods. A total of about 500 terrestrial images was acquired using a CANON EOS 6D camera, featuring lenses with focal lengths of $24 \mathrm{~mm}$ and $35 \mathrm{~mm}$. The images were taken both at the bottom of the Modi and Alyssos rocks, as well as at the top of these rocks, where ruins of old Monasteries and other constructions exist, for their geometric documentation in a higher LoD. They have a size of
$5472 \times 3648$ pixels and a pixel size of $6 \mu \mathrm{m}$. These terrestrial images are complementary to the aforementioned aerial and UAV datasets. In addition, the acquisition of terrestrial laser scans, using a FARO Focus3D X330 laser scanner, at the top of the Modi rock took place. Finally, LiDAR and imagery data were acquired by the dual-channel airborne mapping system RIEGL VQ-1560i-DW. A total number of 23 high resolution images and a point cloud, collected by two LiDAR scans, covered an area of $4 \mathrm{~km}^{2}$. In order to orient the images and the point clouds, the collection of GCPs in the field was required. In this context, 25 GCPs that cover the whole Meteora site and 15 additional GCPs that cover the two rocks were collected via a dual-frequency GNSS device using the real time kinematic (RTK) method and their coordinates in the Greek Geodetic Reference System '87 (GGRS '87) were determined.

\subsection{D Modelling}

The aerial and UAV images were oriented through the automated structure from motion (SfM) technique, using the Agisoft Metashape software (Agisoft, 2020). Its first step is the extraction of tie points in the overlapping images using a variation of the SIFT algorithm (Lowe, 2004). Then, the images are oriented and a sparse point cloud of the scene is generated. The sparse point cloud of the Meteora site, derived by the aerial images, consists of about $3.4 \mathrm{M}$ points. The sparse point cloud of Modi and Alyssos, derived by the UAV and terrestrial images, consists of about 7.4M points.

Then, dense point clouds were generated using Agisoft Metashape through automated dense image matching. The dense point cloud of the Meteora site, which was derived using the oriented aerial images, consists of about $353.1 \mathrm{M}$ points, while the dense point cloud of Modi and Alyssos, generated using the oriented UAV and terrestrial images, consists of about $500 \mathrm{M}$ points.

At a next step, the 3D surface models of the area of interest were automatically created using the Agisoft Metashape software and were manually edited using the Geomagic Studio software (3D Systems, 2020). These 3D models were inserted again into the Agisoft Metashape software for the generation of texture maps, through manual selection of the images. Views of the generated 3D textured models of the Meteora site, as well as for the Modi and Alyssos rocks, are illustrated in Figure 3 and Figure 4, respectively.

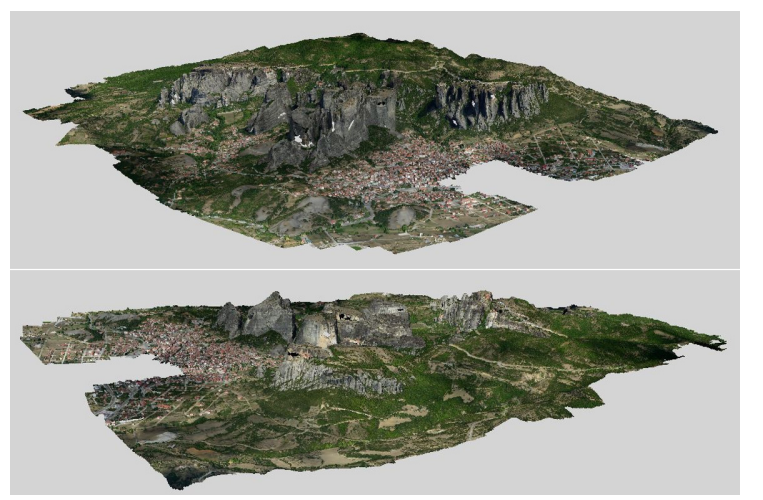

Figure 3. 3D views of the 3D textured model of the Meteora site

The airborne LiDAR system provided independent point cloud data, i.e., one for each channel (green and near-infrared). The point clouds were of high-density $\left(\sim 80\right.$ points $\left./ \mathrm{m}^{2}\right)$ and large volume. Thus, they were organized into subsets of 
$500 \mathrm{~m} \times 500 \mathrm{~m}$. The files were firstly converted to .las format and pre-processing was applied, in terms of sampling and noise removal. Then, the strips were aligned and a quick segmentation for classification and extraction of features was conducted. In order to minimize measurement inaccuracies, the GCPs were used to rectify the point cloud georeferencing. Finally, color mapping was implemented, using the georeferenced RGB images that were captured in parallel with the scans (Figure 5).

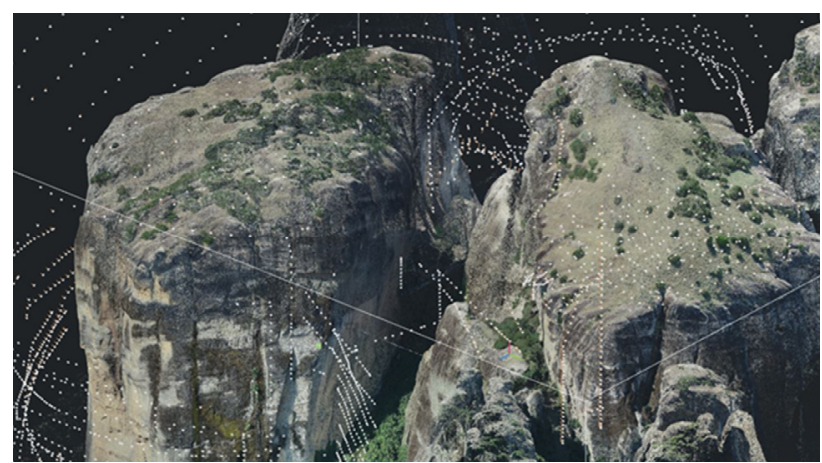

Figure 4. 3D views of the 3D textured models of Modi and Alyssos rocks

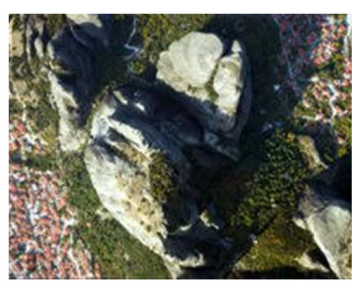

(a)

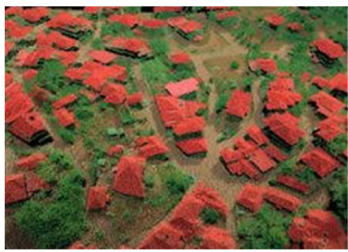

(b)

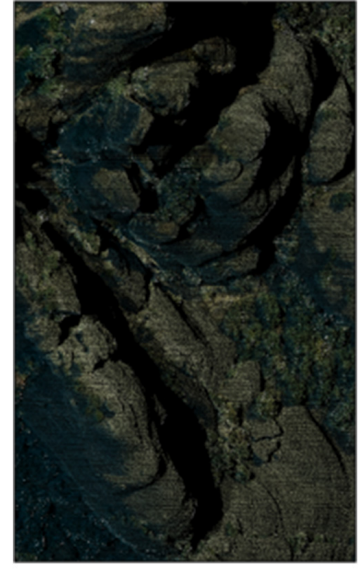

(c)
Figure 5. LiDAR data and processing stages: (a) Aerial image captured by the camera of the airborne mapping system; (b) result of the feature detection and extraction process using the point cloud; (c) Final point cloud with color mapping

\subsection{Assets and Multi-type Data}

Apart from the collection of spatial data, non-spatial data were collected, so that the geometric model of the area of interest can be linked to a database with various kinds of information. The collection of literature of historic interest as well as the study of hagiological, etymological and laographic data took place, in order to create a database incorporating such kind of information, from the establishment of the monastic community in Meteora up to today. Moreover, visits of the project consortium were conducted to local parishes, municipalities and communities, for collecting laographic information and religious data. Also, interviews were conducted with local actors, concerning traditional customs. Furthermore, old photographs, videos, drawings and audio clips were collected from the locals, which further enrich the database material. These are the assets that are used by the METEORA platform for enriching the user experience.

\subsection{D Visualization and AR}

Within the METEORA project, 3D meshes of varying LoDs are generated. These models are used for the creation of a single 4D functional model, which incorporates scale as the fourth dimension. These meshes are generated using the different data that were collected, as described in section 3.1 (Figure 6). The scale dimension will be used not only for displaying the model of the proper resolution, depending on the requirements of each user of the platform, but also for the efficient and seamless visualization, so that points of the model that are closer to the user are depicted in greater detail than distant points, without requiring any action by the user. In addition to single LoD approximations for each model, progressive streaming and refinement will take place, to show significant features and temporal changes. The 4D model may comprise representations of different historic and restoration phases, or incorporate DEMs, CADs, wireframe models or 3D small-scale detailed artefacts.

The AR mechanism superimposes $3 \mathrm{D}$ virtual reconstructions on real heritage assets and places virtual and textual overlays, as well as navigation instructions, in order to display information without interfering with the users' perspective. It is enabled when the integrated location service receives positional data (longitude/latitude) of the area of interest. The user location is known from data obtained by the GPS sensor of the mobile device. Filtering and correction of GPS signals need also to be implemented for obtaining more precise positional information.

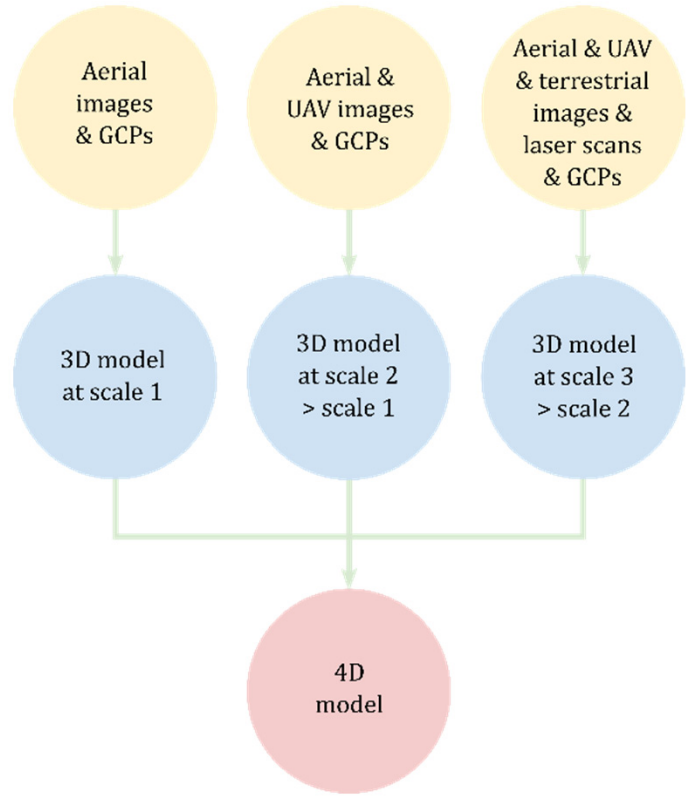

Figure 6. 4D modelling scheme

\section{USER SCENARIOS}

In the context of the METEORA project, we ran a survey to gather some formal, direct evaluation from the users of the platform. The questionnaire consisted of demographic questions, multiple choice questions, rating scale questions and open-ended questions; it included a short investigation about the user background, some questions about the attractiveness of the characteristics of the platform, some specific questions depending on the kind of users, as well as information on the ways of using the platform. The questionnaire was made available to participants both in printed form (e.g. in local 
services, in the Municipality of Trikala and the Municipality of Meteora) as well as via Internet (docs.google.com/forms/d/e/ 1FAIpQLSdNJ18yASzb-hf4Q9oV11KRDp-C-ZRA0VgQTDH YhniKMC2keQ/viewform). It was posted on the Meteora website, on the official pages of the project on social media, in groups of professional associations (e.g., the Hellenic Association of Urban and Regional Planners), as well as in webpages of touristic interest (e.g., the Information Tourist Center of Kalambaka - Infotourist)

In a three-months running period, we received 224 valid responses. These responses were translated into a form that was manipulated to produce statistics. The results showed that 182 questionnaire recipients are interested in the METEORA platform as tourists; 151 recipients are interested as scientists; 47 recipients are interested as business people; and 31 recipients are interested as members of a local authority dealing with cultural heritage (e.g., museums). Each questionnaire recipient had the choice to select multiple answers concerning the use of the METEORA platform. Hence, the sum of the aforementioned responses exceeded the total number of the sample. The categories of users of the METEORA platform were set in the questionnaire; however, the answers of the recipients regarding the way of using the platform, depending on their user category, contributed in formulating the user scenarios, as described in the following. The functionalities of the METEORA platform, regarding its scenarios of use for tourists, will be publicly available to everyone. However, if the users belong to any of the other three user categories (i.e., scientists, business people or members of a local authority dealing with cultural heritage), they will have to log in to the METEORA platform using their credentials; then, the additional functionalities that correspond to their user category will become available to them.

\subsection{Touristic Use}

There are three categories of scenarios of use of the METEORA platform for a tourist: (i) touristic use before visiting the site of interest; (ii) touristic use during the visit to the site of interest; and (iii) touristic use after visiting the site of interest.

4.1.1 Before Visiting the Site of Interest: The METEORA platform can be used by tourists who plan to visit a site of interest, in order to organize their stay and acquire a complete picture of its history and beauties. Also, visitors to the sites can make better use of their time, so they can visit as many places of interest and attractions as possible. In addition to tourists residing in areas other than the place of interest, people everywhere can connect with their place of origin and explore the sights of their place. The scenarios presented in the following can be distinguished for a tourist before visiting a site of interest.

According to the scenario "Virtual tour to the site of interest", the tourists may use the METEORA platform to navigate in the textured 3D model of the site of interest. An important characteristic of the platform is the "supervised navigation" (Figure 7); it is a navigation in various parts of the scene with seamless movements of the 3D model and the camera. By activating the mechanism, successive transitions within the $3 \mathrm{D}$ space from both high altitude and ground height provide the user with a complete understanding of the site of interest. Rotations and changes in viewing angle help to overcome the problem of invisible parts of the scene. At the same time, information is automatically displayed about the projected landmark or toponym in a text field above the scene. The sync of the display and the corresponding information provides a clear and critical overview of the individual points of interest. The tourists can choose among the available alternative routes of supervised navigation to virtually navigate in the site of interest. In addition, they may freely navigate in the available 3D model of the site of interest, with the possibilities to rotate, pan, zoom in/out and interact with points on the map and in the navigation menu. Besides, the METEORA platform supports 4D models (section 3.4). This means that when the user navigates in the $3 \mathrm{D}$ model, its resolution is constantly being optimized, depending on the distance of the navigation camera, i.e., the scale of the model automatically changes as the user moves on it. The scale is differentiated both in the case of supervised navigation and in the case of free navigation.

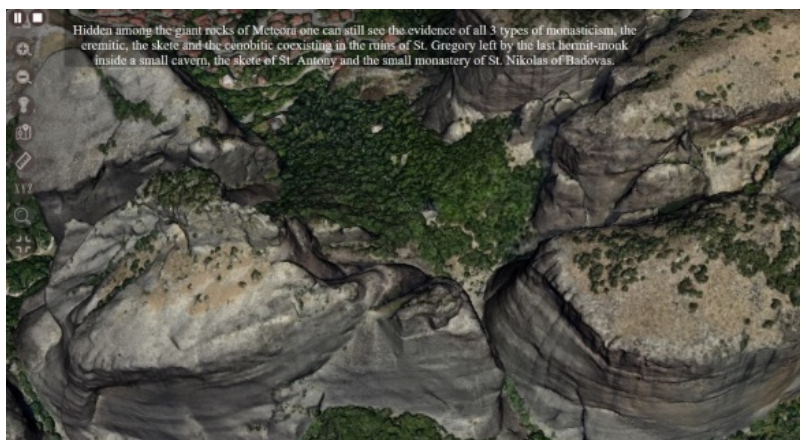

Figure 7. Screenshot from supervised navigation in the Meteora site via the METEORA platform

According to the scenario "Virtual tour to the site of interest and search for information", in addition to the virtual tour to the place of interest mentioned in the previous scenario, the tourists - users of the METEORA platform - are additionally interested in information about the site of interest, such as its history, religious information, accommodation information, leisure, etc. Thus, as they navigate in the $3 \mathrm{D}$ model of the place of interest, at specific points of the model manually selected by them, dynamic information is presented in the form of text or multimedia (e.g., images/videos). When the users locate a landmark or point of interest, the METEORA platform indicates that information is available about it. If selected, the corresponding image or textual information is displayed (Figure 8). Unlike conventional systems, these are not just text notes or simple labels. They include links to navigate in the web page and the digital collections of the platform. The links transform the linear and passive information provision into an interactive experience and the relationship between the elements of the METEORA platform is transformed from spatial to logical. Before redirecting to any page, the users are able to choose whether or not to visit it.

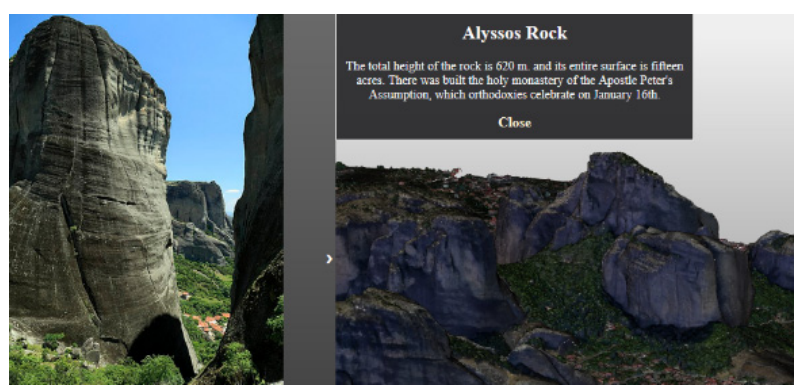

Figure 8. Screenshot from the display of text and image data for Alyssos rock via the METEORA platform 
According to the scenario "Search for information about the site of interest", the tourists - users of the METEORA platform - are interested in learning as much as possible about the history, folklore, manners and customs of the place to be visited, while not navigating in the photorealistic 3D model of the site of the visit or before/after navigating in it. In addition, they are interested in looking for points of interest on the map (Figure 9) and learning information about them. The METEORA system provides support for dynamic content search tables, allowing the users to search for information of interest. Dynamic lookup table data are extracted from the system database. The interactive options for dynamic tables are related to partial and cumulative search functions using filters. The data are in the form of a list of categories allowing the visitors to perform combinatorial queries. The users can perform simple data searches (partial search) or develop complex searches using combinatorial queries (cumulative search).

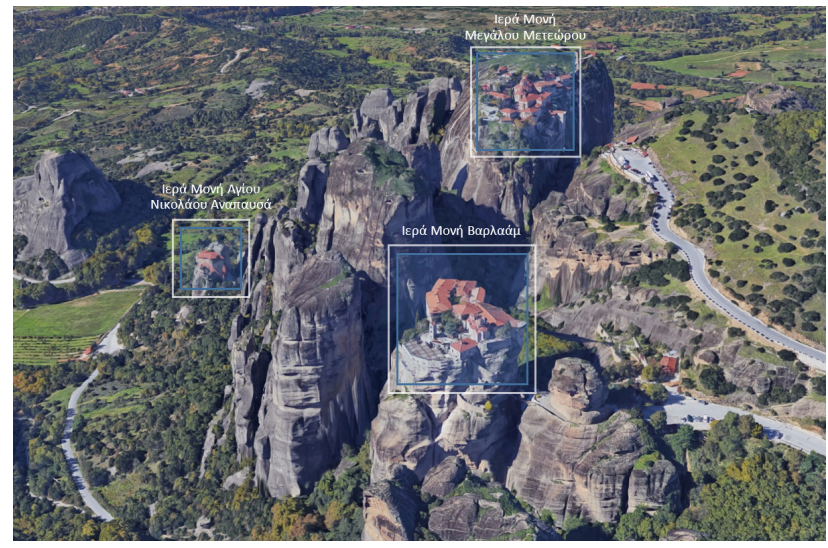

Figure 9. Search for points of interest on the map via the METEORA platform

4.1.2 During the Visit to the Site of Interest: The METEORA platform can be used via a mobile device (tablet, mobile phone) by tourists during their visit to a place of interest, under one or more of the scenarios presented in the following.

According to the scenario "Localized virtual tour", the platform can be used by tourists during their visit to the site of interest for a localized virtual tour in an area that is inaccessible or difficult to be accessed. The platform detects the location of the users through the GPS sensor of their mobile device; it enables them to navigate to nearby areas of interest and displays suggestions for sightseeing tours. As far as the localized virtual navigation is concerned, as mentioned in section 4.1.1, it can be either supervised or free.

According to the scenario "Augmented Reality", tourists, during a visit to a site of interest, can make use of the AR function of the platform, through a mobile device, for enrichment of the surrounding landscape with additional elements/information related to the earlier form or future restoration of the site. These additional elements may be either 3D models of the current or future status of sites of interest, or information that is superimposed on the users' view of the real world in the proper place, in the form of text or images or other multimedia (graphics, video, audio).

According to the scenario "Detection of alternative activities and points of interest", the METEORA platform can be used by tourists during a visit to a site of interest to identify alternative activities in the wider area of the site and in nearby points of interest. The platform locates their location through the GPS sensor of their mobile device and displays activities and points of interest in the wider area where the tourists are located.

4.1.3 After Visiting the Site of Interest: The METEORA platform can also be used by tourists after their visit to a place of interest. During their visit to a site of interest, it is possible that they were not able to visit some places of interest, because these places were either inaccessible or difficult to be accessed. Hence, according to the scenario "Virtual tour in inaccessible/difficult-to-be-accessed areas", the METEORA platform enables the users to navigate to such areas that they did not manage to visit. As mentioned in section 4.1.1, the navigation can be either supervised or free.

Also, according to the scenario "Detection of the next site to visit", the tourists can search through the METEORA platform for the next place to visit. Through virtual navigation in the various sites included in the METEORA platform and by searching for information about them, the tourists can choose the next destination, after being informed about it.

\subsection{Scientific Use}

In order to group the specialties of the scientists-users of the platform and form the corresponding user scenarios, the following categories of users that may utilize the METEORA platform for scientific reasons are distinguished: (i) geotechnical engineers; (ii) archaeologists/architects; (iii) historians/philologists/theologians; and (iv) teachers.

4.2.1 Geotechnical Engineers: Geotechnical engineers (e.g., civil/surveying engineers), may use the METEORA platform to either see details of the 3D structural models of sites of interest and study their geometry via the platform or download the corresponding models/point clouds that are available through the platform and process them offline in the office. According to the first scenario, geotechnical engineers may navigate freely in the 3D model of interest and zoom in details of it that they want to study (see also section 4.1.1, scenario "Virtual tour to the site of interest"). Moreover, the second scenario is very useful, as the system provides such scientists with spatial data of interest that may be further processed by them for scientific reasons.

4.2.2 Archaeologists/Architects: Architects/archaeologists may use the METEORA platform to see details of the 3D models of sites of interest and study their geometry through free navigation (see also section 4.1.1, scenario "Virtual tour to the site of interest"). Furthermore, such scientists could see additional data for a specific model, while navigating in it, e.g., $3 \mathrm{D}$ models of the interior space of a monument and archaeologic or architectural information in the form of text or images, as illustrated in Figure 10 (see also section 4.1.1, scenario "Virtual tour to the site of interest and search for information").

4.2.3 Historians/Philologists/Theologians: Human scientists dealing with history, philology and theology may use the METEORA platform in order to be informed about the history, folklore, manners and customs of a place as well as for religious or other cultural heritage information, either while navigating in the textured 3D model, as shown in Figure 11 (see also section 4.1.1, scenario "Virtual tour to the site of interest and search for information") or without a simultaneous navigation (see also 
section 4.1.1, scenario "Search for information"). Another example of use of the METEORA platform is the display of "hotspots" superimposed on a 3D model, i.e., the display of points of historic/religious interest through graphical labeling.

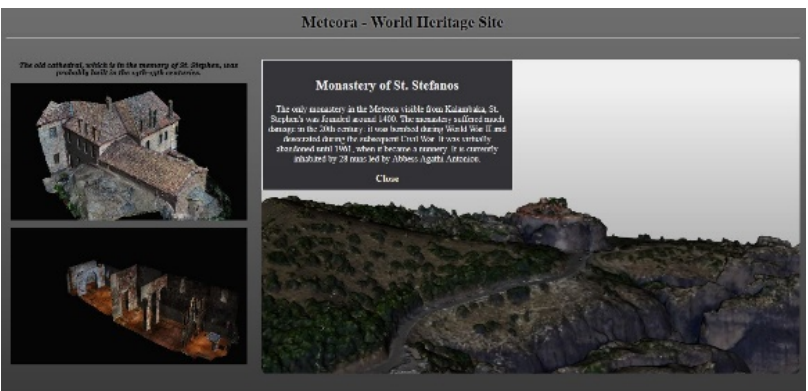

Figure 10. Screenshot from the display of text and 3D models of archaeologic and architectural interest via the METEORA platform

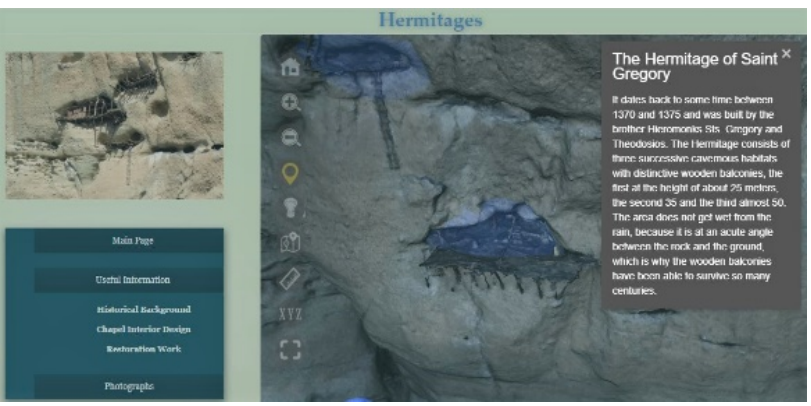

Figure 11. Screenshot from the display of historic and religious information via the METEORA platform

4.2.4 Teachers: The METEORA platform may be a very useful tool for teachers to teach their students about the history of a site, religious and archaeological information about it, as well as the traditions and customs of a historic place of interest. The students will be able to learn such information for the place of interest through an easy and interactive process. Through a virtual tour to a historic place of interest from the desktop PCs of their school, they will be able to see the geometry of the place and additionally search interactively for other kinds of information (see also section 4.1.1, scenario "Virtual tour to the site of interest and search for information").

\subsection{Business Use}

The METEORA platform may be professionally used both by business people in the tourism/commerce zone and by tour guides, as stated in the following.

\subsubsection{Business people in the Tourism/Commerce Zone:} Business people in a touristic/commerce zone (e.g., owners of a restaurant/café, owners of a mini market) that is near a site of interest included in the METEORA platform may use the latter to improve their business profile and increase its competitiveness. This may be achieved through the inclusion of their business in the database of the METEORA platform. In this way, each time tourists, users of the platform, try to identify alternative activities in the wider area of the site where they are located through a mobile device, according to the scenario "Detection of alternative activities and points of interest" (section 4.1.2), their business is displayed as a nearby point of interest.
4.3.2 Tour Guides: The METEORA platform may be used as a tool for touristic tours by tour guides, who provide tourists with information about the place they are visiting and guide them in their visits to places of interest. Guides may encourage tourists to perform the scenarios "Localized virtual tour" or "Augmented Reality" (section 4.1.2) so that they make their tour more interactive and interesting.

\subsection{Cultural Expert Use}

The METEORA platform may be used by members of a local authority dealing with cultural heritage (e.g., museums) to promote the cultural heritage through a modern and innovative platform, upgrade the cultural electronic services and attract a new generation of tourists and visitors, by integrating culture into the tourism experience and promoting the tourism product, while at the same time promoting the history and the culture of a site. Additionally, the METEORA platform may be used by the members of an authority dealing with cultural heritage to promote the work conducted by the authority. For instance, all these may be achieved through inclusion in the METEORA platform of the 3D model of the interior space of a church of historic and archaeological interest, or plans of restoration or old photographs of a cultural site, which are under the jurisdiction of a local authority. Additionally, the METEORA platform may be used by visitors of such a cultural authority (e.g., museum) for an interactive experience (e.g., virtual tour, see also section 4.1.1).

\section{CONCLUSIONS AND FUTURE WORK}

The presented work refers to the ongoing METEORA research project and analyses its scope and methodology up to current results. The objective of the project is the multi-dimensional documentation of a complex archaeological site, like the Modi and Alyssos rocks of the Meteora area, and the integration of the outputs in an appropriately structured database and visualization schema, in order to create an information system that can handle multiple data of heterogeneous types (both geospatial and non-spatial data). It aims at documenting not only the two rocks but also at correlating and disseminating scientific, cultural and archaeological information of the whole archaeological site. This paper presents each stage of the entire workflow: from multi-source data collection, processing and defining the components of the platform to presentation of the system architecture, design principles and use cases. The final implementation involves the creation of a $4 \mathrm{D}$ web visualization platform, with a series of assorted data and information services. It supports a relational database management system, 4D modelling, progressive streaming, AR functionalities and real-time manipulation of the $3 \mathrm{D}$ scene. Its interactive access and retrieval tools parse the cultural resources for specific purposes, like conservation, condition monitoring, education, research and tourism. They foster and endorse studies conducted by a variety of disciplines like natural and physics sciences, humanities, engineering, or activities undertaken by non-professionals.

Many features remain to be implemented and evaluation tests remain to be conducted in order to develop the final version of the METEORA platform and draw final conclusions. The proposed combination has to demonstrate adequate robustness, in order to handle big heritage data of great heterogeneity. At the current phase, additional field work and optimizations to the prototype need to be made. Specifically, the following steps will be conducted within the METEORA project: 
(i) Incorporation of more multi-type data in the prototype platform: LiDAR point cloud, 3D virtual reconstructions of the old religious monuments that existed on top of the two rocks, 3D prominent assets or detailed parts of small-scale and high resolution.

(ii) Development of innovative continuous loading and rendering techniques for the $4 \mathrm{D}$ visualization infrastructure.

(iii) Investigation of the possibility of the platform to handle big data, so that it incorporates multiple data from several case study areas, in addition to Meteora.

(iv) Investigation of the increase of the automation of the $3 \mathrm{D}$ modelling process for areas and monuments of high complexity, especially in the steps of selecting images for texture mapping and automating masking.

(v) Functional testing of the back-end system and performance monitoring to detect failure points, bottlenecks and other potential issues simulating realistic network conditions.

\section{ACKNOWLEDGEMENTS}

This research has been co-financed by the European Union and Greek national funds through the Operational Program Competiveness, Entrepreneurship and Innovation, under the call RESEARCH-CREATE-INNOVATE (project code: T1EDK02859).

\section{REFERENCES}

A-Frame, 2020. A-Frame. www.aframe.io (9 April 2020).

Agisoft, 2020. Agisoft Metashape Software. www.agisoft.com (9 April 2020).

Agugiaro, G., Remondino, F., Girardi, G., von Schwerin, J., Richards-Rissetto, H., De Amicis, R., 2012. A web-based interactive tool for multi-resolution 3D models of a Maya archaeological site. Int. Arch. Photogramm. Remote Sens. Spatial Inf. Sci., XXXVIII-5/W16, 23-30. doi.org/10.5194/ isprsarchives-XXXVIII-5-W16-23-2011.

Ai, B., Wang, L., Yang, F., Bu, X., Lin, Y., Lv, G., 2019. Continuous-scale 3D terrain visualization based on a detailincrement model. ISPRS Int. J. Geo-Inf., 8(10), 465. doi.org/ 10.3390/ijgi8100465.

AR.js, 2020. AR.js - Augmented Reality on the Web. www.arjs-org.github.io/AR.js-Docs/ (9 April 2020).

Ben Ellefi, M., Drap, P., Papini, O., Merad D.D., 2019. Ontology-based web tools for retrieving photogrammetric cultural heritage models. Int. Arch. Photogramm. Remote Sens. Spatial Inf. Sci., XLII-2/W10, 31-38. doi.org/10.5194/isprsarchives-XLII-2-W10-31-2019.

Cao, M., Wang, P., Wu, L., Lu, Z., Lu, Q., 2018. The research on the online publishing platform of point clouds of Chinese cultural heritage based on LIDAR technology: A case study of Chen Clan Academy in Guangzhou, Guangdong Province. IOP Conf. Ser.: Mater Sci. Eng., 452:032019. doi.org/10.1088/1757899X/452/3/032019.

Cipriani, L., Bertacchi, S., Bertacchi G., 2019. An optimised workflow for the interactive experience with cultural heritage through reality-based 3D models: Cases study in archaeological and urban complexes. Int. Arch. Photogramm. Remote Sens. Spatial Inf. Sci., XLII-2/W11, 427-434. doi.org/10.5194/isprsarchives-XLII-2-W11-427-2019.

Dall' Asta, E., Bruno, N., Bigliardi, G., Zerbi, A., Roncella, R., 2016. Photogrammetric techniques for promotion of archaeological heritage: The archaeological museum of Parma (Italy). Int. Arch. Photogramm. Remote Sens. Spatial Inf. Sci., XLI-B5, 243-250. doi.org/10.5194/isprsarchives-XLI-B5-2432016.

Draco, 2020. Draco. www.google.github.io/draco (9 April 2020).

El-Mahgary, S., Virtanen, J.-P., Hyyppä, H., 2020. A Simple Semantic-Based Data Storage Layout for Querying Point Clouds. ISPRS Int. J. Geo-Inf., 9(2), 72. doi.org/10.3390/ ijgi9020072.

Ioannidis, C., Piniotis, G., Soile, S., Bourexis, F., Boutsi, A.M., Chliverou, R., Tsakiri, M., 2019. Laser and multi-image reverse engineering systems for accurate $3 \mathrm{D}$ modelling of complex cultural artefacts. Int. Arch. Photogramm. Remote Sens. Spatial Inf. Sci., XLII-2/W11, 623-629. doi.org/10.5194/ isprs-archives-XLII-2-W11-623-2019.

Ioannidis, C., Verykokou, S., Soile, S., Potsiou, C., 2015. 5D multi-purpose land information system. Eurographics Workshop on Urban Data Modelling and Visualisation, Delft, The Netherlands, pp. 19-24. doi.org/10.2312/udmv.20151344.

López, L., Torres, J.C., Arroyo, G., Cano, P., Martín, D., 2019. An efficient GPU approach for designing 3D cultural heritage information systems. J. Cult. Herit., 41, 142-151. doi.org/ 10.1016/j.culher.2019.05.003.

Ohori, K.A., Ledoux, H., Biljecki, F., Stoter, J., 2015. Modeling a $3 \mathrm{D}$ city model and its levels of detail as a true 4D model. ISPRS Int. J. Geo-Inf., 4(3), 1055-1075. doi.org/10.3390/ ijgi4031055.

Potenziani, M., Callieri, M., Dellepiane, M., Corsini, M., Ponchio, F., Scopigno, R., 2015. 3DHOP: 3D Heritage Online Presenter. Computers \& Graphics, 52, 129-141. doi.org/ 10.1016/j.cag.2015.07.001.

Salonia, P., Scolastico, S., Pozzi, A., Marcolongo, A., Messina, T.L., 2009. Multi-scale cultural heritage survey: Quick digital photogrammetric systems. J. Cult. Herit., 10, e59-e64. doi.org/10.1016/j.culher.2009.09.004.

Three.js, 2020. Three.js. www.threejs.org (9 April 2020).

van Oosterom, P., Meijers, M., 2011. Towards a true varioscale structure supporting smooth-zoom. 14th ICA/ISPRS Workshop on Generalisation and Multiple Representation, Paris.

van Oosterom, P., Meijers, M., 2014. Vario-scale data structures supporting smooth zoom and progressive transfer of 2D and 3D data. Int. J. Geogr. Inf. Sci., 28(3), 455-478. doi.org/ $10.1080 / 13658816.2013 .809724$

3D Systems, 2020. Geomagic Studio. www.3dsystems.com (9 April 2020) 